\title{
Hybridization of desalination processes
}

\author{
Mohamed M.Elmesmary ${ }^{1}$, Abdulwahab J. Alsultan ${ }^{2}$ \\ ${ }^{1}$ (The Higher Institute of Energy Water Resource Department, The Public Authority for Applied Education and \\ Training, \\ ${ }^{2}$ (The Higher Institute of Energy Water Resource Department, The Public Authority for Applied Education and \\ Training,
}

\begin{abstract}
The aim of any new or improved technique in the water production technology may concentrate in water quality , production rates and coasts of production .In this paper we will talk about the hybridization of desalination process intending to enhance the effectiveness of the productionprocedure.. Hybridization of a dependable production process such as Multi-Stage Flash (MSF) and a well improved and introduced technology such as Membrane-based process is an upcoming trend for maximizing the recovery ratio and quality for the process, in addition to the economical benefits that it will offer. This paper will discuss examples of different hybrid systems.
\end{abstract}

Keywords: Hybridization, Desalination, Multi stage Flash, Membrane, Water

\section{INTRODUCTION}

In the last few decades and due to population increase and other natural demands the consumption rate of potable water increased worldwide. In GCC countries the demand for fresh water increased while there are so limited natural fresh water sources; the water production depends entirely in industrial production process. This fact lead to search for new fresh water production technologies to sustain such growing demands. This improvement has been restricted to certain criteria's such as the quantity produced of fresh water, the quality of production, the dependability of the process and further more the cost of water production.

In this paper, some of the main processes of desalination are over looked to illustrate the concept of Hybridization of desalination processes; which is the combination of different desalination systems in a certain configuration to produce fresh water. Cogeneration of power and fresh water is a well known concept by now, and hold for so many benefits economical wise, an integration of dual purpose plants with a hybrid system will support the aims of constructing such plants.

Hybridization of a dependable production process such as Multi-Stage Flash (MSF) and a well improved and introduced technology such as Membrane-based process is an upcoming trend for maximizing the recovery ratio and quality for the process, in addition to the economical benefits that it will offer. Studies have been conducted to demonstrate these influences of Hybridization on water production plants.

\section{SINGLEPRODUCTION PROCESSES}

Water production processes such as MultiStage Flash Evaporation (MSF) and Multi-Effect Distillation (MED) are considered conventional main systems used to produce fresh water in large scales for different domestic and industrial use . Typical MSF system may contain between 24 to 30 stages that would give a production capacity of 450 to $57000 \mathrm{~m}^{3} /$ day.

MSF systems will have the advantage of high production rates with high reliability but with relatively high the installation cost.

Multi-Effect Distillation (MED) is to be considered as an evaporative process in large scale. Multiple effects in series running at lower pressure are used where the vapor from an effect is used to vaporize water in the subsequent effect. High Gain Ratio which means to produce more water per amount of steam used is one of the advantages of this system beside its High efficiency. In aspect of production rates, production rates of MED plants are lower than MSF plants production rates.

Other main production technique is the Reverse Osmosis, there is no phase change in the Membrane-based process, which consumes only electric energy used mainly to provide the pumping power needed for the operation. RO systems use different types of semi-permeable membranes . Appling pressure to the feed water that is greater than its osmotic pressure separate dissolved salts from water and this produces fresh water through the membrane.

The energy consumption in membranebased process is proportional to the concentration of dissolved salts in the feed water. RO systems allows for tolerated salt concentration in the feed water and 
that is the most tolerant desalination process concerning salt concentration. It is to be noted that RO plants reliability can be increased with proper pre-treatment of feed water.

In most of the recent statistics it can be said that water recovery in $\mathrm{RO}$ plants may reach the percentage of 35 to $50 \%$.

To achieve higher production ratios , large number of membranes will be needed and this will increase the initial cost of the plant and later on will increase the membranes replacement costs.

A high ratio of fresh water to electrical power demands emphasize the trend of using DualPurpose Plants, which cogenerate potable water side by side with electrical power. In MSF and MED plants.

\section{HYBRIDIZATION}

Hybridization can be explained as the Integration of thermal desalination technologies with membrane desalination technologies. Hybrids systems in which permeate from a Membrane-based process such as $\mathrm{RO}$ desalination component is mixed with distillate from thermal desalination component. The reduction of initial and production coasts is one of the main objectives of integrating different technologies and another main goal is to reduce the water production overall costs.

Several Hybrid systems are developed during the last few decays which offers a improved and promising performance. The recovery increase in fresh water from saline sources is widely investigated as an indication of improved performance of Hybrid system over conventional single process systems. Furthermore, due to the high share of pre-treatment in the total cost of desalination, the increase of recovery may really improve the economical performance of desalination to a great extent.

\section{CHARACTERISTICS OF HYBRID SYSTEMS}

Hybrid Systems can offer various advantages over conventional thermal desalination processes. It can be said that the recovery of water using RO (Reverse Osmosis) is higher than using MSF ( Multi-Stage Flash) and MED (Multi-Effect Distillation). In general, sea water reverse osmosis system (SWRO) recovery rates ranges from 30 to $45 \%$ of the feed water while the other portion is returned back to the sea. While other systems like MSF and MED would give recovery ratio ranges from 12 to $20 \%$.

Major advantages of the RO systems maybe surmised in the fast and easy Start up and shut dawn procedures beside the relatively easy running and operation process. Use of RO system and MSF system means that fresh water is treated twice, the water in integrated plants can be used twice but it needs to be treated once leading to the reduction of the pre-treatment process and cost .The integration of RO system and MSF system in one process enables better and effective control of the feed water temperature going into the RO plant by means of using the warm reject coolant water from the heat rejection section of the MSF system. By using this technique, water production of the RO system is improved.

The most common set up for Hybridization especially in GCC region is the MSF-RO Hybrid Desalination System, which is discussed in the next section.

\section{SET UP FOR HYBRIDIZATION SYSTEMS}

In GCC countries MSF desalination technology is the most commonly used commercial water production techniques from seawater. However, in the last decays RO desalination technology participation in water production in GCC countries are increasing every year due to its advantages and increasing demands .

Integrating both MSF system and RO system introduces a hybrid system known as MSF-RO Hybrid desalination System . Integration between MSF system and RO system can be done in different configurations according to the technology used but general speaking this integration leads to many benefits in both technical aspects and economic or cost aspects.

By using hybrid MSF-RO desalination System it is normally expected to have increased product water recovery same time it is also expected to achieve lower specific energy consumption for water production .It will be also expected to have lower consumption of chemicals used in the production process, with RO membrane replacement rates reduced and life cycle of these RO membranes are also prolonged.

In figure (1) showing one of the simplest MSFRO Hybrid configuration which can be configured and utilized to an existing MSF plants. The configuration explain a system that using the cooling seawater rejected from the MSF heat rejection section to feed the RO desalination system. The distillate produced from the MSF plant is then blended with permeate from the RO plant process producing the final water for different consumption purposes . 


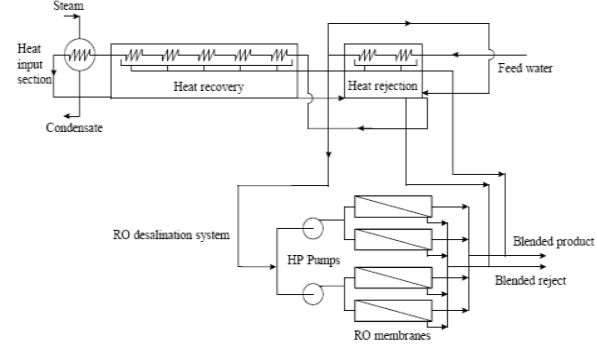

Figure (1): example of basic MSF-RO hybrid system

\section{HYBRID MSF/RO SYSTEM}

Hybridization of seawater reverse osmosis (SWRO), desalting technology and the multi-stage flash (MSF) has been considered in many literatures as a case study to improve the performance of the most common MSF process and also to reduce the cost of the desalted water. Coupling of the two processes could be made on different level of integration. Optimization studies were conducted to study different configurations of MSF-RO Hybrid System. Some of the studies were based on the goal of achieving maximum overall plant recovery which is defined as the ratio between the mass flow rate of the combined product and the total mass flow rate of seawater feed to the plant including cooling water to the ejector condenser. Other studies are based on the aim of studying means of achieving minimum water production cost obtained from the design and cost calculations of each configuration.

As mentioned in the previous section, hybridization of MSF and RO systems could be introduced in so many configurations. A.M. Hellal [ 3 ] discussed some of these configurations in trend to study the optimal design based on economical considerations. In order to construct a mathematical modeling of Hybrid MSF-RO configuration, Equations to describe MSF and RO plants process is derived. additional and/or modified equations are introduced to describe each case of hybridization configuration.

Another approach was introduced by $\mathrm{E}$. Cardona, A. Piacentino and F. Marchese [4], different methods and techniques were explained aiming to higher efficiency and in same time reducing the cost of fresh water desalination plants .one of the approaches was integrating different desalination systems into a hybrid mechanical and thermal configuration. And the most expected gains to be achieved by hybrid systems are reduction in pretreatments requirements and costs, reduction of overall cost and important energy savings.

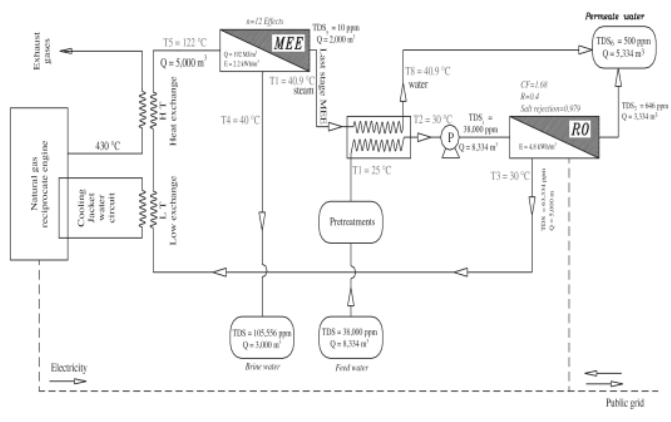

Fig. 2. Simple hybrid desalination plant

E. Cardona, A. Piacentino and F. Marchese [4] studied a simple system with configuration of a small size (2000 m3/day) thermal desalination system (MEE) which is combined with a singlestage seawater reverse osmosis system (SWRO). The studied system used a natural gas reciprocate engine, the exhaust gases are used for heat recovery together with heat recovery from the cooling jacket water circuit. The study aimed to cover different sides of the configuration such as technical, energy, and cost saving aspects.

In this case study, the hybrid system showed economically convenient performance with respect to the production of the same fresh water flow rate by very efficient RO systems. The aim of reducing the overall cost and minimizing the unit cost of desalted water was discussed during the performed optimization. Another factor discussed was the effect of fresh water unit cost with the variations in the flow rate of permeate exiting the RO section to reach for the optimal design and configuration.

Another model concentrated in studying a system configuration using forward osmosis (FO) combined with other processes such as RO or evaporation because the draw salts in the FO products should be removed. The purpose of using forward osmosis system was due to its lower energy consumption to produce water than reverse osmosis (RO) .

This model was presented by JuneSeokChoia, Hwan Kimb, SanghoLeea*, TaeMunHwanga, HyunjeOha, DaeRyookYangc, Joon Ha Kimd [6]. The study paper investigated the theoretical different points of the combined system with FO and RO for seawater desalination. Studying the performance of the combined systems showed that the combinations of both forward osmosis system with seawater reverse osmosis may lead to achieving a high recovery rate reaching up to $80 \%$. It was also found that forward osmosis system may be integrated or combined with brackish reverse osmosis system to produce high quality of water at higher rates and higher recovery compared to conventional RO based desalination systems. 


\section{ECONOMICAL ASPECTS}

The configuration in (Fig.3) shows another example of the saving study by Leon Awerbuch [ 8 ] using a configuration of 100 MIGD $(455,000 \mathrm{~m} 3 / \mathrm{d})$ MSF desalination system and $400 \mathrm{MW}$ of electric power generation plant. The estimated annual fuel cost requirement listed in the study mentioned a figure of 86 million US \$ based on historic fuel cost of only $1.1 \mathrm{US} \$ / \mathrm{GJ}$ considered during the study.

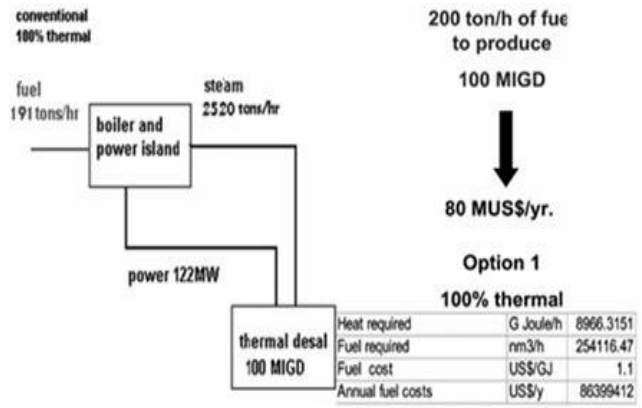

Fig. 3 A case study of the thermal + RO plant configuration

Based on financial assumption during the study period, and Assuming a system with $60 \%$ thermal and $40 \%$ RO hybrid 100 MIGD $(455,000$ $\mathrm{m} 3 / \mathrm{d}$ ) desalination plant ,this configuration will operate at reduced fuel consumption of only 55 million US\$ per year. This annual fuel cost difference of over 30 million US\$ per year is based on 1.1 \$/GJ, considering the impact of assumed fuel price of 10 . $\$ / G J$ the annual cost differential will exceed 300 million dollars .This amount of saving can lead to pay back for the total capital expenditure in time interval of 3 years.

\section{CONCLUSIONS}

Developing hybrid systems by Combining thermal and membrane desalination processes and technologies within a single plant schemes will lead to the reduction of overall water production costs, further more it will add flexibility to the combined water and power production process and reduce any existing water and power demand mismatch problems

It can be confirmed that applying such solutions will reduce desalinated water costs, compared with other conventional schemes, from as little as $2-3 \%$ to as much $15 \%$. In large desalination plants, there should also be little loss of economies of scale due to the use of two or more different processes, in two or more smaller units, instead of one large single technology plant.

Hybrid systems of MED-RO has many of the same advantages than the systems of MSF-RO, but has the advantage of significantly power water (PWR) ratio reduction .
Further studies needed to demonstrate the ideas that improved Hybrid systems such as MSFMED with VC and Hybrid with NanofiltrationSoftening Membrane has the potential of boosting water output through simple or full integration and at the same time reduces power to water (PWR) ratio and increase desalination output of distillation plants MSF and MED, by reducing scaling potential of the feed, increase the top brine temperature and provide significant better concentration factors and recovery for all distillation processes.

Hybrid with electrically driven desalination technologies RO and VC would allow use offpeak power for water production, and minimize power capacity by shutting down RO or VCdaily during the peak.

\section{REFERENCES}

[1]. AsamAlmulla, Ahmad Hamad. Integrating hybrid systems with existing thermal desalination plants. Desalination, 174 (2005) 171-192.

[2]. Sergei P. Agashichev, M.E. El-Dahshan. Reverse osmosis incorporated into existing cogenerating systems as a sustainable technological alternative for UAE. Desalination, 157 (2003) 33-49.

[3]. A.M. Helal, A.M. El-Nashar, E. AlKatheeri, S. Al-Malek. Optimal design of hybrid RO/MSF desalination plants Part I: Modeling and algorithms. Desalination, 154 (2003) 43-66

[4]. E. Cardona , A. Piacentino, F. Marchese Performance evaluation of CHP hybrid seawater desalination plants- Department for Energy and Environmental ResearchesFaculty of EngineeringUniversita` di Palermo

[5]. Ennio Cardona, Salvatore Culotta, Antonio Piacentino, Energy saving with MSF-RO series desalination plants. Desalination, 153 (2002) 167-171.

[6]. June-SeokChoia, Hwan Kimb, SanghoLeea Theoretical investigation of hybrid desalination system combining reverse osmosis and forward osmosis Korea Institute of Construction Technology, 2311 Daehwa-Dong

[7]. Raphael Semiat. Desalination: Present and Future. International Water Resources Association Water International, Volume 25, Number 1, Pages 54.65, March 2000

[8]. Leon Awerbuch President, Leading Edge Technologies, Ltd., Winchester, MA USA, Integrated Hybrid Desalination Systems 